Supplementary Information

\title{
CL6mN: Rationally Designed Optogenetic Photoswitches with Tunable Dissociation Dynamics
}

Abhirup Mukherjee**1, Chaitanya Sudrik**2, Yuge Hu**1, Manish Arha ${ }^{3}$, Mark Stathos ${ }^{4}$, Jieung Baek $^{5}$, David V. Schaffer*5,6,7, Ravi S. Kane*1

\section{Affiliations:}

${ }^{1}$ Department of Chemical and Biomolecular Engineering, Georgia Institute of Technology, Atlanta, GA 30332

${ }^{2}$ Department of Chemical and Biological Engineering, Rensselaer Polytechnic Institute, Troy, NY 12180

${ }^{3}$ Centre for Biotechnology and Interdisciplinary Studies, Rensselaer Polytechnic Institute, Troy, NY 12180

${ }^{4}$ Bioengineering Graduate Program, Georgia Institute of Technology, Atlanta, GA 30332

${ }^{5}$ Department of Bioengineering, University of California, Berkeley, Berkeley, California, 94720, USA.

${ }^{6}$ Department of Chemical Engineering, University of California Berkeley, Berkeley, California, 94720, USA.

${ }^{7}$ Helen Wills Neuroscience Institute, University of California, Berkeley, Berkeley, California, 94720, USA.

This file includes:

Supplementary Figure S1: Characterization of the ability to activate Wnt signaling in CL6mNtransfected cells.

Supplementary Figure S2: Characterization of the effect of CL6m5 on non-canonical Wnt pathways.

Supplementary Figure S3: Comparison of fluorescence intensity for cells transfected with Cry2PHR and CL6mNs.

Supplementary Figure S4: Characterization of number of clusters for cells transfected with Cry2PHR and CL6mNs normalized with respect to the cellular area.

Supplementary Figure S5: Characterization of dissociation half-life for Cry2PHR-GFP. 


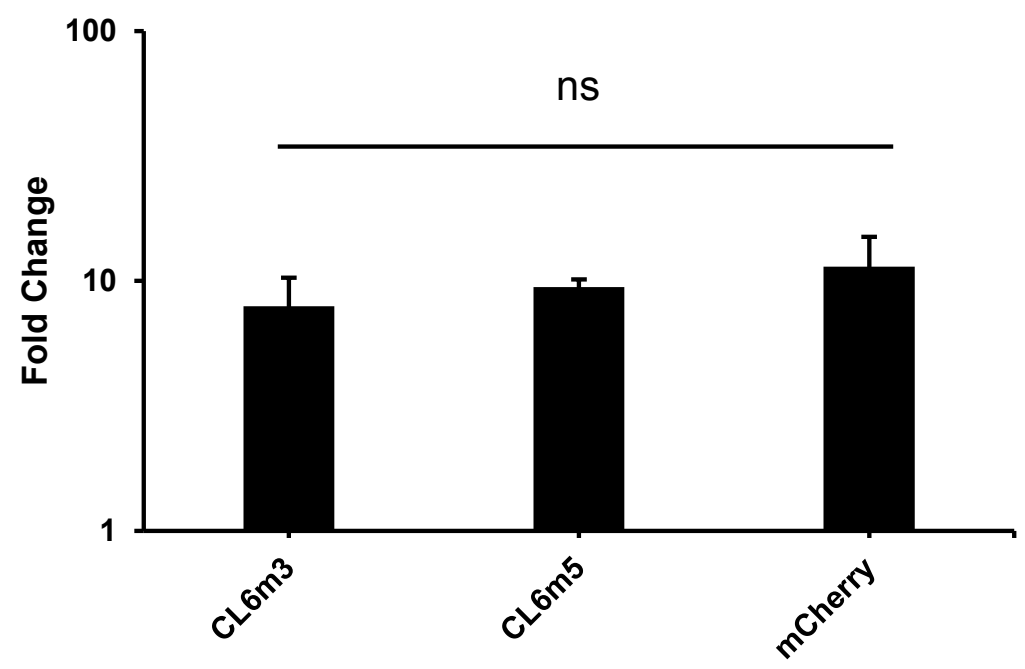

Supplementary Figure S1. Characterization of the ability to activate Wnt signaling in CL6mNtransfected cells. A luciferase assay was used to probe the activation of canonical Wnt signaling. Data are normalized relative to those for cells not treated with Wnt-3a. Significant activation was observed for cells transfected with plasmids encoding CL6m3, CL6m5, or mCherry (control) when treated with $15 \mathrm{nM}$ commercial Wnt-3a ligand compared to untreated cells. However, there was no significant difference in the extent of activation between groups (ns: not significant, $\mathrm{n}=3$ replicates, One-way ANOVA) 


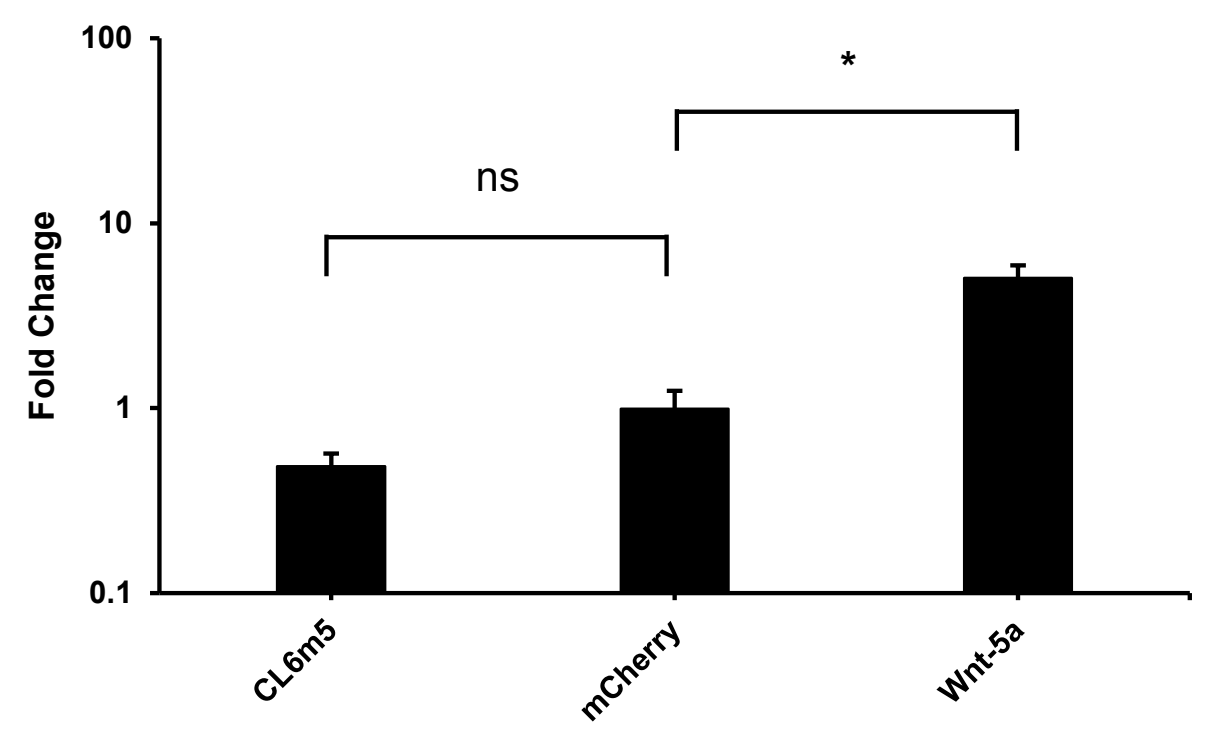

Supplementary Figure S2. Characterization of the effect of CL6m5 on non-canonical Wnt pathways. A luciferase assay was used to probe the activation of non-canonical Wnt signaling. While activation was seen for cells transfected with plasmids encoding Wnt-5a, no significant activation was observed in cells transfected with plasmids encoding CL6m5 (relative to controls: cells transfected with plasmids encoding mCherry). ( $* \mathrm{p}<0.05$, ns: not significant $\mathrm{n}=3$ replicates, One-way ANOVA with Tukey post hoc test) 

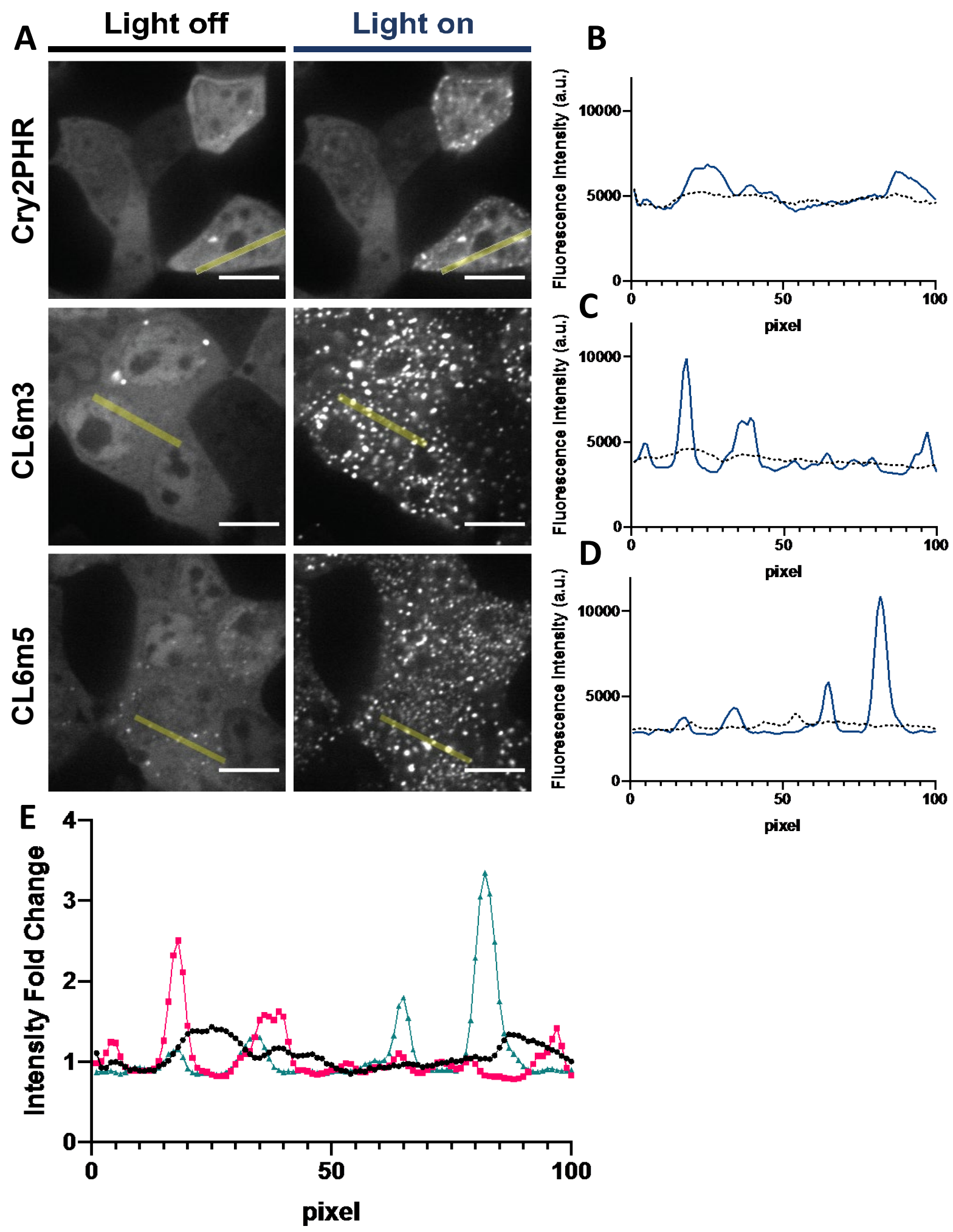

Supplementary Figure S3. Comparison of fluorescence intensity upon clustering of Cry2PHR and CL6mNs. (A) Confocal images of cells transfected with Cry2PHR (top panel), CL6m3 (middle panel), and CL6m5 (bottom panel) before and after exposure to 488-nm laser pulses. Fluorescence intensities from pixels labeled with yellow are plotted in (B), (C), and (D) for light 
off (black dashed line) and light on (blue solid line) for Cry2PHR, CL6m3 and CL6m5 respectively. (E) Fold change based on intensities in Cry2PHR (black circles), CL6m3 (red square) and CL6m5 (green triangle) light-on samples normalized against the average fluorescence intensities in light-off samples. Scale bars in (A), $10 \mu \mathrm{m}$. 

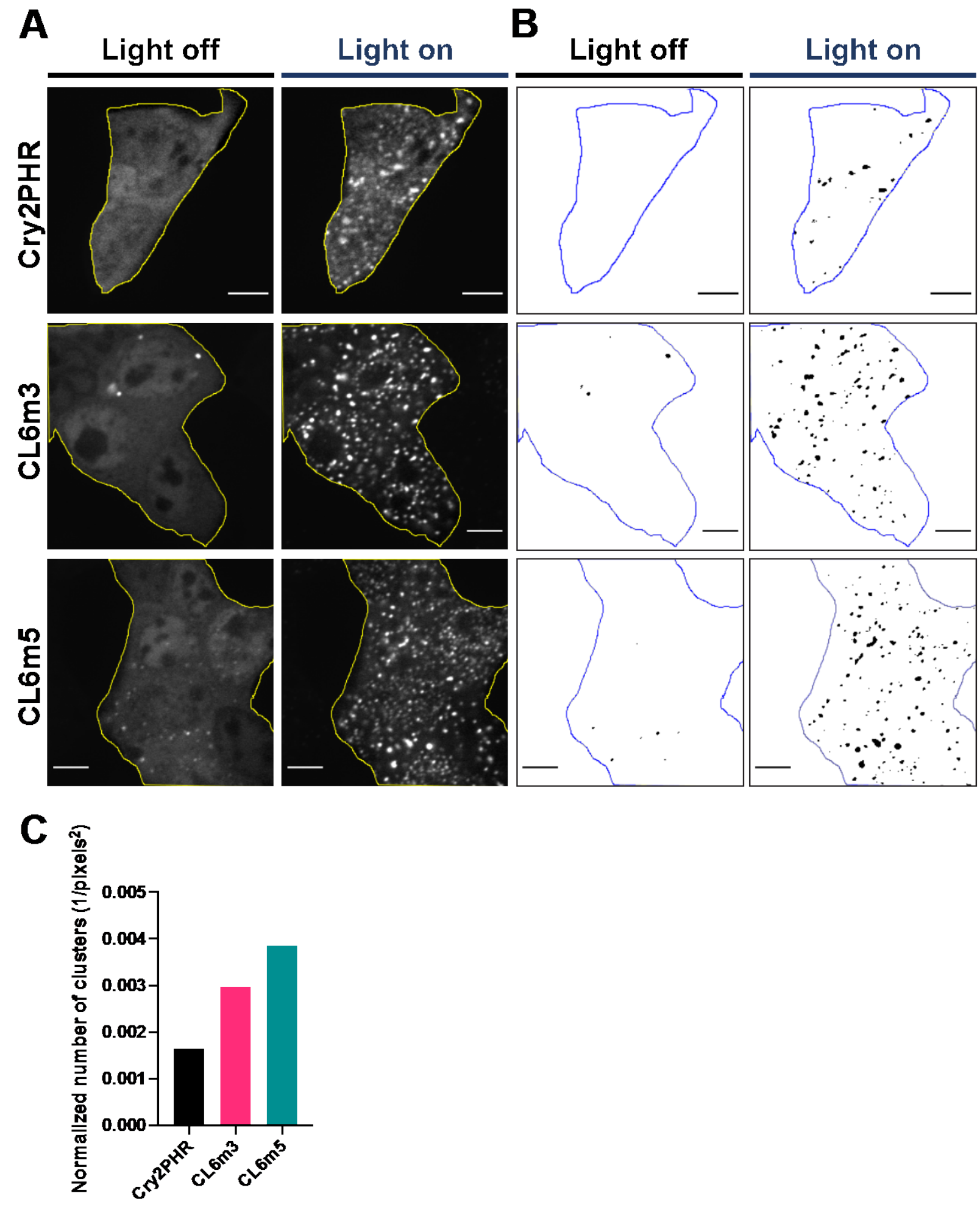

Supplementary Figure S4. Characterization of number of clusters of Cry2PHR and CL6mNs normalized with respect to the cellular area in a representative image. (A) Comparison of light on 
and light off samples for Cry2PHR and CL6mNs with selected areas (bounded by yellow lines). Image contrasts are auto-set by FIJI based on light-on samples. (B) Comparison of thresholding results of light-on and light-off samples of Cry2PHR and CL6mNs. Same selected areas are bounded by blue lines as (A). Same thresholding cutoff was applied to both light-on and light-off samples of each construct so that the resulting thresholding light-off results are reflective of the original light-off confocal images with auto contrast in (A). (C) Number of clusters obtained by "Analyze Particles" after thresholding in FIJI normalized over selected area. Scale bars in (A) and (B), $6 \mu \mathrm{m}$. 


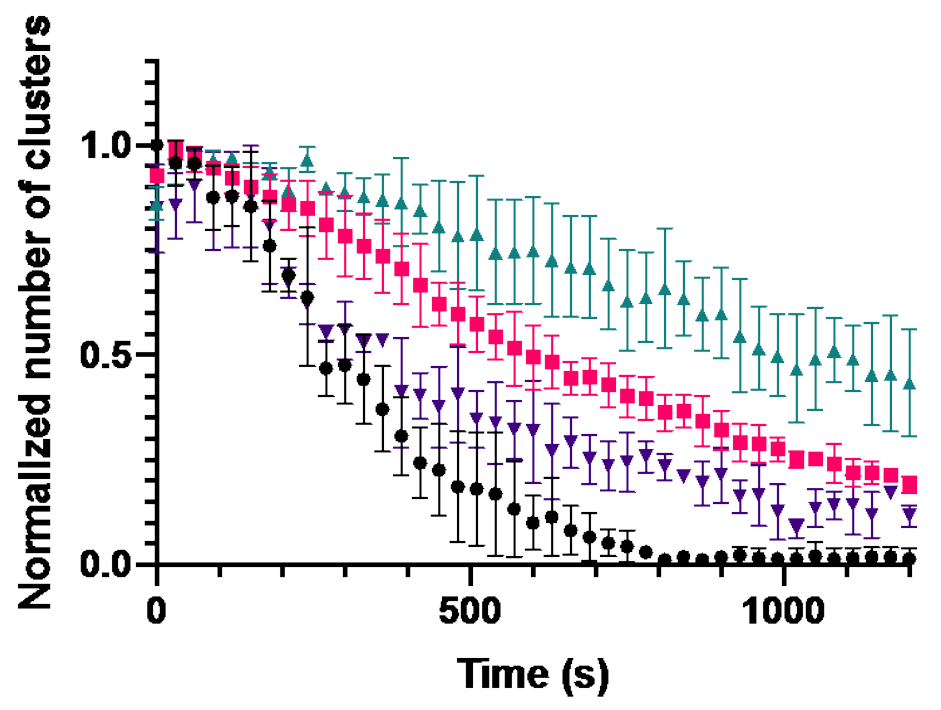

Supplementary Figure S5. Characterization of dissociation half-life at $25^{\circ} \mathrm{C}$ for Cry2PHRGFP. Comparison of declustering dynamics for Cry2PHR (black dots), Cry2PHR-GFP (purple triangles), CL6m3 (pink squares), and CL6m5 (green triangles). Cry2PHR-GFP has a dissociation half-life of 5.9 minutes, shorter than that for CL6m3 and CL6m5. 